\title{
Prognostic value of echocardiographic and Doppler parameters in horses admitted for colic complicated by systemic inflammatory response syndrome
}

\author{
Laura Borde, DVM, PhD, DECEIM; Hélène Amory, DVM, PhD, DECEIM; Sigrid Grulke, DVM, \\ PhD, DECVS; Aurélia A. Leroux, DVM, MS; Rosa M. Houben, DVM; Johanne Detilleux, DVM, PhD \\ and Charlotte C. Sandersen, DVM, PhD, DECEIM
}

\begin{abstract}
Objective - To assess the prognostic value of echocardiographic parameters of left ventricular (LV) function in horses with systemic inflammatory response syndrome (SIRS).

Design - Prospective observational study.

Setting - Veterinary teaching hospital.

Animals - Forty-one horses admitted for colic with clinical evidence of SIRS.

Interventions - All horses underwent Doppler echocardiographic examination on admission. LV echocardiographic parameters, including pulsed-wave tissue Doppler imaging parameters, were compared between nonsurvivors $(n=29)$ and horses that survived to discharge $(n=12)$.

Measurements and Main Results - With comparable heart rate and LV preload estimate, LV stroke volume index, the velocity time integral, deceleration time, ejection time of Doppler aortic flow, and peak early diastolic myocardial velocity were lower in the nonsurviving than in the surviving horses, while pre-ejection period to ejection time ratio (PEP/ET) of Doppler aortic flow and the peak early diastolic filling velocity to peak early diastolic myocardial velocity ratio $(\mathrm{E} / \mathrm{Em})$ were higher $(P<0.05)$. A cut-off value of 0.26 for PEP/ET predicted mortality with $100 \%$ sensitivity and $42 \%$ specificity (area under the receiver operating characteristic curve: 0.71 ), whereas a cut-off value of 2.67 for E/Em predicted mortality with $100 \%$ sensitivity and $83 \%$ specificity (area under the receiver operating characteristic curve: 0.89 ).

Conclusions - Echocardiography may provide prognostic information in colic horses with clinical evidence of SIRS. Especially, PEP/ET and E/Em could be useful markers of systolic and diastolic dysfunction, respectively, to detect horses with a high risk of death requiring more intensive cardiovascular monitoring as it has been reported in human patients with septic shock.
\end{abstract}

(J Vet Emerg Crit Care 2014; 24(3): 302-310) doi: 10.1111/vec.12177

Keywords: Endotoxemia, equine intensive care, hemodynamics, prognosis

\begin{tabular}{l}
\hline Abbreviations \\
CI $\quad \begin{array}{l}\text { cardiac index } \\
\text { CO }\end{array}$ \\
cardiac output \\
From the Department of Clinical Sciences, Faculty of Veterinary Medicine, \\
University of Liège, Boulevard de Colonster 20, B41, 4000 Liège, Belgium. \\
The authors declare no conflict of interest. \\
Presented in part at the 4th Congress of European College of Equine Internal \\
Medicine, Hannover, Germany. \\
Adress correspondance and reprint requests to \\
Dr. Laura Borde, Department of Clinical Sciences, Equine Internal Medicine, \\
Faculty of Veterinary Medicine, University of Liège, Boulevard de Colonster \\
20, B41, 4000 Liège, Belgium. E-mail: lauraborde@hotmail.fr \\
Submitted October 31, 2012; Accepted February 04, 2014.
\end{tabular}

\section{Abbreviations}

2D

EDV

$\mathrm{E} / \mathrm{Em}$

Em

$\mathrm{EF}$

ET

ETI

HR

IVRT

LV

MODS two-dimensional mode

left ventricular end-diastolic volume peak early left ventricular filling velocity to peak early diastolic myocardial velocity ratio peak early diastolic myocardial velocity left ventricular ejection fraction left ventricular ejection time left ventricular ejection time index corrected for heart rate

heart rate isovolumetric relaxation time left ventricle multiple organ dysfunction syndrome 


\begin{tabular}{|c|c|}
\hline PEP & left ventricular pre-ejection-period \\
\hline PW-TDI & pulsed-wave tissue Doppler imaging \\
\hline SD & standard deviation \\
\hline SI & stroke volume index \\
\hline SIRS & systemic inflammatory response syndrome \\
\hline SS & shock score \\
\hline $\mathrm{Vcf}$ & $\begin{array}{l}\text { mean velocity of circumferential fibre short- } \\
\text { ening }\end{array}$ \\
\hline $\mathrm{Y}$ & Younden index \\
\hline
\end{tabular}

\section{Introduction}

Endotoxemia is a common and potentially fatal complication of acute intestinal disease in adult horses. ${ }^{1}$ Endotoxic shock, like septic shock, involves hypovolemic, cardiogenic, and distributive components resulting in tissue hypoperfusion $^{2}$ with a high risk for multiple organ dysfunction syndrome (MODS) and increased mortality in equine $^{1,3}$ and human ICUs. ${ }^{4,5}$ As a result, most clinical and laboratory parameters currently used as prognostic indicators of colic in adult horses are related to cardiovascular status. ${ }^{6}$

Endotoxins stimulate a systemic inflammatory response syndrome (SIRS), which is associated with severe hemodynamic disturbances via vasoplegia, a drastic reduction in venous return and myocardial depression causing a drop in systemic vascular resistance and cardiac output (CO). ${ }^{7,8}$ Sympathetic stimulation and release of various endogenous substances provide a compensatory vasoconstriction and an increase in heart rate (HR), myocardial contractility and venous return to preserve CO. However, loss of vascular responsiveness to catecholamines and myocardial depression finally contribute to a progressive drop in $\mathrm{CO}$ and systemic vascular resistance despite persistent tachycardia. ${ }^{2,4,7}$

A shock score (SS) combining several clinical and laboratory parameters on admission enabled prediction of survival in colic horses but could not discriminate, which severely affected horses with shock were likely to respond to treatment. ${ }^{6}$ A comparable "Mortality in Emergency Department Sepsis Score," based on clinical and laboratory variables is currently considered inaccurate in predicting mortality in people with severe sepsis or septic shock, ${ }^{9}$ especially in the presence of tissue hypoperfusion associated with a high risk of MODS. ${ }^{10}$ Hemodynamic monitoring is required in these patients to predict the response to fluid therapy, the need for vasopressors or inotrope drugs and outcome. ${ }^{5,11,12}$ A poor prognosis has been associated with persistent tachycardia along with low systemic vascular resistance, ${ }^{11}$ a refractory response to catecholamines, ${ }^{13}$ and slow lactate clearance or low central venous oxygen saturation postresuscitation. ${ }^{14}$ In critically ill horses, lactate clear- ance after resuscitation recently emerged as a promising marker of systemic hypoperfusion with some prognostic value. ${ }^{15,16}$

Although frequently observed in people with septic shock, myocardial failure is rarely the cause of death in these patients who succumb mostly from MODS. ${ }^{17} \mathrm{Nev}-$ ertheless, serum concentrations of cardiac biomarkers (ie, cardiac tropinin $\mathrm{T}_{1}^{18}$ amino-terminal pro-brain natriuretic peptide) ${ }_{1}^{19}$ and echocardiographic and Doppler parameters of left ventricular (LV) systolic and diastolic function proved to be strongly associated with outcome in people with septic shock. ${ }^{4,5,11,12,20-22}$

A moderate increase in serum cardiac tropinin I in the postoperative period has also recently been associated with a poor outcome in 34 horses undergoing emergency laparotomy. ${ }^{23}$ A previous echocardiographic study has suggested a systolic dysfunction in colic horses with clinical signs of endotoxic shock. ${ }^{24}$ To date however, the prognostic value of echocardiographic parameters is unknown.

The aim of this prospective study was to investigate the value of two-dimensional (2D), Motion-Mode (M-mode), Doppler, and pulsed-wave tissue Doppler imaging (PW-TDI) echocardiography in predicting the outcome of colic in adult horses with clinical signs and laboratory parameters compatible with SIRS.

\section{Material and Methods}

\section{Study population}

Horses referred between March 2009 and September 2011 for colic with clinical and laboratory parameters compatible with SIRS at admission were included in the study (Table 1). The presence of SIRS was assumed when horses were hyperlactatemic (plasma lactate > $2 \mathrm{mmol} / \mathrm{L})$ or had low systolic arterial pressure $(<$ $90 \mathrm{~mm} \mathrm{Hg}$ ) and fulfilled at least 2 of the following criteria: 1. hypo- or hyperthermia (rectal temperature $<36.7^{\circ} \mathrm{C}$ or $\left.>38.6^{\circ} \mathrm{C}\right)$, 2. leukopenia or leukocytosis (WBC < $5,000 / \mu \mathrm{L}$ or $>14,500 / \mu \mathrm{L}), 3$ ) tachycardia (HR $>50 / \mathrm{min}$ ) or 4 ) tachypnea (respiratory rate $>25 / \mathrm{min}$ ). Horses euthanized for financial considerations, immature horses ( $<2$ years old), or horses that recieved $\alpha 2$-receptor agonists before echocardiographic examination were excluded from the study.

\section{Clinical data and blood analysis recording}

HR, respiratory rate, and rectal temperature were recorded at admission for each horse. Jugular vein EDTA blood samples were obtained and immediately analyzed. Lactate concentration was measured using a portable device ${ }^{\mathrm{a}}$ and $\mathrm{WBC}$ was determined using an automatic analyzer. ${ }^{b}$ Systolic arterial pressure was 
Table 1: Comparison of the means $\pm \mathrm{SD}$ of the parameters used to determine the presence of systemic inflammatory response syndrome in 12 surviving and 29 nonsurviving adult horses admitted for colic. Least-squares-mean test was significant if $P<$ 0.05 (ANOVA model including breed, body weight, age, sex, and outcome)

\begin{tabular}{lccl}
\hline & $\begin{array}{l}\text { Survivors } \\
\boldsymbol{n}=\mathbf{1 2}\end{array}$ & $\begin{array}{l}\text { Nonsurvivors } \\
\boldsymbol{n = 2 9}\end{array}$ & $\boldsymbol{P}$-value \\
\hline Heart rate (per min) & $65.64 \pm 18.43$ & $79.71 \pm 26.52$ & 0.0818 \\
Respiratory rate $($ per $\mathrm{min})$ & $27.1 \pm 10.6$ & $30.7 \pm 14$ & 0.1544 \\
WBC $($ count/ $\mu \mathrm{L})$ & $12.01 \pm 4.62$ & $9.31 \pm 5.32$ & 0.26 \\
Rectal temperature $\left({ }^{\circ} \mathrm{C}\right)$ & $37.62 \pm 0.65$ & $37.8 \pm 0.66$ & 0.6135 \\
Plasma lactate & $3.57 \pm 3.67$ & $3.43 \pm 2.28$ & 0.9939 \\
$\quad$ concentration $(\mathrm{mmol} / \mathrm{L})$ & & & \\
SAP $(\mathrm{mm} \mathrm{Hg}) *$ & $94.7 \pm 0$ & $122.9 \pm 26$ & - \\
\hline
\end{tabular}

SAP: systolic arterial pressure (noninvasive); *insufficient data for statistical analysis: only available in 9 horses including only 1 survivor.

measured noninvasively by a manometer equipped with an occlusion cuff ${ }^{c}$ placed over the coccygeal artery.

\section{Echocardiography}

Each horse underwent echocardiographic examination on admission. All echocardiographic records were performed and analyzed by the same operator (LB) using a portable echocardiograph ${ }^{\mathrm{d}}$ equipped with a 1.5-3.6 $\mathrm{MHz}$ transducer. It permitted echocardiographic view numeric recording (still frames or cine-loops) with a simultaneous continuous single lead base-apex ECG for delayed analysis. All examinations were recorded digitally and analyzed offline. ${ }^{\mathrm{e}}$

Echocardiographic recordings and measurements were obtained conforming to conventional methods. ${ }^{25-27}$ Assessment of aorta and cardiac chamber sizes was performed using standard transthoracic 2D and M-mode echocardiography. The following standard views were recorded for analysis: a right parasternal 2D long-axis 5 chamber view, a TM short-axis view of the LV at the level of the chordae tendinae, and a left parasternal 2D long-axis 4 chamber angled view.

Assessment of LV wall motion was obtained from the standard left parasternal short-axis view of the LV at the level of the chordae tendinae by placing a sample volume of $5.9 \mathrm{~mm}$ in the endocardial region of the LV free wall in diastole with the PW-TDI mode. Special attention was paid to keeping the sample volume on the myocardium during all phases of the cardiac cycle. The velocity scale was set to $-30 \mathrm{~cm}$ to $+20 \mathrm{~cm} / \mathrm{s}^{28}$

Assessment of aortic flow was performed using standard transthoracic PW-Doppler echocardiography. Transaortic flow velocity profiles were obtained from the standard left parasternal long axis 5-chamber view in PW-Doppler mode by placing the sample volume $(4.91 \mathrm{~mm})$ downstream from the aortic valve in the aortic root. ${ }^{29-31}$

Transmitral flow velocity profiles were recorded by positioning the transducer as ventrally as possible and angling dorsally from the standard left parasternal long axis 4-chamber view in PW-Doppler mode with the sample volume placed between the opened tips of the mitral leaflets in diastole. ${ }^{30,31}$

Systolic function was assessed by means of fractional shortening (FS = [LVIDd-LVIDs]/LVIDd) and ejection fraction (EF: difference between end-diastolic and endsystolic volume) derived from 2D and M-mode views, completed by measurement of systolic time intervals on pulsed-wave-Doppler aortic flow profiles. Although these parameters reflect systolic function including myocardial contractility, all of them are influenced by HR and LV loading conditions. Pre-ejection period (PEP) was measured from the onset of the QRS complex to the onset of systolic aortic flow. PEP estimates the isovolumetric contraction time or the time needed to generate enough pressure within LV to open the aortic valve. Aortic ejection time (ET) was measured from the onset to the end of aortic flow. ET typically decreases with reduced preload (low volume) or force of contraction. ET index (ETI = $\mathrm{ET}+0.55 \times \mathrm{HR}$ ) and pre-ejection period to ejection time ratio (PEP/ET) were calculated to reduce the influence of HR on systolic time intervals. Velocity of circumferential fiber shortening integrates the ET to the fractional shortening equation $(\mathrm{Vcf}=\mathrm{FS} / \mathrm{ET})$ and represents how fast the ventricle shortens. ${ }^{27}$

Diastolic function was assessed by means of transmitral flow velocity profiles in pulse-wave Doppler mode, associated with isovolumetric relaxation time (IVRT) and peak early diastolic myocardial velocity (Em) derived from PW-TDI mode. Diastolic dysfunction may result from impaired or delayed relaxation or from reduced LV compliance. Peak early LV diastolic filling (E) corresponds to the rapid ventricular filling in early diastole opposite to peak late LV ventricular filling resulting from atrial contraction (A). Combined to IVRT (time from closure of aortic valve to onset of filling with opening of mitral valve), E/A ratio permits to estimate the filling pressure gradient between left atrium and LV. They are affected by preload, myocardial relaxation and HR. E peak depends on LV relaxation rate and filling pressure during early diastole (influenced by left atrial pressure, LV preload, succion, and compliance). Since Em translates LV relaxation rate, peak early left ventricular filling velocity to peak early diastolic myocardial velocity ratio $(\mathrm{E} / \mathrm{Em})$ reflects $\mathrm{LV}$ filling pressure during early diastole, typically increased in case of impaired ventricle compliance resulting in a restrictive ventricular filling. ${ }^{27}$ 
In case of impaired relaxation (but also with tachycardia or a falling preaload), the relaxation continues until the late diastolic filling phase and the atrial contribution increases in the ventricular filling leading to a prolonged IVRT associated with a low E/A ratio on the mitral flow profile combined with a low Em on PWTDI. Conversely, a restrictive ventricular filling leads to a normal or shorter IVRT associated to high E/A and $\mathrm{E} / \mathrm{Em}^{27}$

\section{Statistical analysis}

For statistical analysis, horses were separated into 2 groups according to outcome: nonsurvivor or survived to discharge. Means and SDs were calculated for each group and for each studied parameter. Some parameters were corrected for body weight before statistical analysis (index = variable/body weight). After verifying that breed, sex, and outcome were independent (Pearson chisquare test of independence), a series of ANOVA models were used to test whether echocardiographic and Doppler parameters were statistically different across the 2 groups, after adjusting for the effects of breed, sex, weight, and age. The ANOVA model was as follows:

$$
y_{i j k l}=\mu+C_{i}+B_{j}+S_{k}+b_{1} W_{i j k l}+b_{2} A_{i j k l}+e_{i j k l},
$$

where $y$ was the echocardiographic or Doppler parameter on the $m$ th horse with survival $C_{i}(i=0,1)$, breed $B_{j}$ $(j=1,2, \ldots, 5)$, sex $S_{k}(k=1,2,3)$, weight $\left(W_{i j k l}\right)$ and age $\left(A_{i j k l}\right), \mu$ was the overall mean, $b_{1}$ and $b_{2}$ are the linear regression effect of $W_{i j k l}$ and $A_{i j k l}$ on $y_{i j k l}$, and $e_{i j k l}$ was the residual term, assuming independance between error terms. The null hypothesis of no difference between ordinary least-squares means for the survival was tested.

The ability of each parameter to predict survival was assessed by calculating the area under the receiver operating characteristic (ROC) curve and the Younden index for each significant echocardiographic parameter (logistic procedure) separately. The cut-off value offering the highest Younden index was retained for each of these parameters with their corresponding sensitivity and specificity. A commercial statistical software package ${ }^{f}$ was used for all statistical analyses. Statistical significance was set at $P<0.05$.

\section{Results}

Forty-one horses fulfilled the inclusion criteria during the study period. Each horse had an SS of at least 3 of 4 according to criteria set by Grulke et $\mathrm{al}^{6}$ (ie, $\mathrm{HR}>80 / \mathrm{min}, \mathrm{RR}>35 / \mathrm{min}, \mathrm{PCV}>55 \%$, systolic arterial pressure $<90 \mathrm{~mm} \mathrm{Hg}$ or plasma lactate $>2 \mathrm{mmol} / \mathrm{L}){ }^{6}$ Animals included were 20 geldings, 17 mares, and 4 stallions. Breed distribution was 31 Warmbloods, 1 Ara- bian, 1 Spanish Breed, 1 Frisian, and 7 riding ponies. Mean \pm SD age was $11.1 \pm 6.6$ years (range: $2-27$ years) and mean \pm SD body weight was $484.5 \pm 78.7 \mathrm{~kg}$ (range: $270-620 \mathrm{~kg}$ ). Twelve horses survived to discharge from the hospital. One horse died spontaneously at induction of anesthesia. Twenty-eight horses were subjected to euthanasia, either early after admission due to a poor surgical prognosis (15 horses), or during the postoperative period (13 horses) because of reocurrence of colic (8 horses) or multiple organ failure (5 horses).

Clinical and laboratory parameters were not significantly different between survivors versus nonsurvivors horses (Table 1). Echocardiographic studies for data collection (3 views recording) did not exceed 15 minutes and were easily integrated into the admission protocol during the time allowed for rectal palpation and abdominal ultrasound. Values for mean \pm SD echocardiographic parameters derived from $2 \mathrm{D}$-mode obtained in the 2 groups and results of the ANOVA test are shown in Table 2. Although the influence of body weight was significant for several morphological echocardiographic variables including end-diastolic and end-systolic LV internal diameter, LV end-diastolic volume (EDV) and end-systolic volume (ESV), none of these variables were significantly different between the 2 outcome groups (survivors versus nonsurvivors). The EDV and ESV indexes corrected for body weight (respectively, EDVI and ESVI), LV fractional shortening and LV EF did not differ significantly between survivors and nonsurvivors.

Values for mean \pm SD Doppler parameters of systolic function obtained in each group and the results of the ANOVA test are shown in Table 3. CO, cardiac index (CI), as well as maximal velocity (Vmax), pre-ejectionperiod (PEP), acceleration time and acceleration rate of aortic flow ejection, were not significantly different between the two groups. However, the velocity time integral, deceleration time and ET of aortic flow, stroke volume, stroke index (SI), and HR-corrected ET (ETI) were significantly lower, and the PEP to ET ratio (PEP/ET) as well as the mean velocity of circumferential fiber shortening (Vcf) were significantly higher in nonsurvivors compared to survivors.

The area under the ROC curve was 0.73 for SI, 0.76 for ET, 0.74 for ETI, and 0.71 for PEP/ET. A cut-off value of $1.78 \mathrm{~mL} / \mathrm{kg}$ for SI predicted mortality with $83 \%$ sensitivity and $62 \%$ specificity (Younden index $(Y)=0.56$ ). A cut-off value of $340 \mathrm{~ms}$ for ET predicted mortality with $75 \%$ sensitivity and $69 \%$ specificity $(Y=0.44)$. A cut-off value of $377 \mathrm{~ms}$ for ETI predicted mortality with $75 \%$ sensitivity and $69 \%$ specificity $(Y=0.44)$. A cut-off value of 0.26 for PEP/ET predicted mortality with $100 \%$ sensitivity and $42 \%$ specificity $(Y=0.41$; Figure 1$)$.

Values for mean \pm SD Doppler parameters and PWTDI parameters of diastolic function obtained in each 
Table 2: Comparison of the means \pm SD values of $2 \mathrm{D}$ and $\mathrm{M}-$ mode echocardiographic parameters in 12 surviving and 29 nonsurviving adult horses admitted for colic complicated by systemic inflammatory response syndrome. Least-squares-mean test was significant if $P<0.05$ (ANOVA model including breed, body weight, age, sex, and outcome)

\begin{tabular}{lccc}
\hline & \multicolumn{1}{c}{$\begin{array}{l}\text { Survivors } \\
\boldsymbol{n}=\mathbf{1 2}\end{array}$} & $\begin{array}{l}\text { Nonsurvivors } \\
\boldsymbol{n}=\mathbf{2 9}\end{array}$ & $\boldsymbol{P}$-value \\
\hline Ao $(\mathrm{cm})$ & $7.8 \pm 0.82$ & $7.55 \pm 0.68$ & 0.0818 \\
IVSd $(\mathrm{cm})$ & $3.61 \pm 0.48$ & $3.61 \pm 0.57$ & 0.7191 \\
IVSs $(\mathrm{cm})$ & $4.6 \pm 0.49$ & $4.52 \pm 0.56$ & 0.8162 \\
LVIDd $(\mathrm{cm})$ & $9.26 \pm 1.29$ & $8.84 \pm 1.55$ & 0.4835 \\
LVIDs $(\mathrm{cm})$ & $5.71 \pm 1.24$ & $5.34 \pm 1.26$ & 0.2900 \\
LVFWd $(\mathrm{cm})$ & $3.09 \pm 0.61$ & $3.08 \pm 0.65$ & 0.7371 \\
LVFWs $(\mathrm{cm})$ & $4.24 \pm 0.52$ & $4.34 \pm 0.57$ & 0.5883 \\
LA $(\mathrm{cm})$ & $10.95 \pm 1.27$ & $10.27 \pm 1.37$ & 0.1827 \\
EDV $(\mathrm{mL})$ & $489.95 \pm 152.72$ & $447.16 \pm 167.06$ & 0.5353 \\
EDVI $(\mathrm{mL} / \mathrm{kg})$ & $0.991 \pm 0.318$ & $0.905 \pm 0.287$ & 0.4142 \\
ESV (mL) & $177.35 \pm 88.11$ & $148.64 \pm 70.43$ & 0.2371 \\
ESVI (mL/kg) & $0.355 \pm 0.175$ & $0.3 \pm 0.134$ & 0.2154 \\
$\% F S(\%)$ & $38.4 \pm 7.94$ & $39.73 \pm 9.9$ & 0.3784 \\
EF $(\%)$ & $65.55 \pm 9.12$ & $66.81 \pm 11.18$ & 0.4399 \\
\hline
\end{tabular}

Ao: aortic diameter; EDV: LV end-diastolic volume; EDVI: LV end-diastolic volume index $=E D V /$ body weight; EF: LV ejection fraction; ESV: left ventricular end-systolic volume; ESVI: ESV index = ESV/body weight; \%FS: fractional shortening; IVS: interventricular septum thickness; LA: left atrium; LV: left ventricular; LVFW: LV free wall thickness; LVID: LV internal diameter; n: number of horses; s: end-systolic; d: end-diastolic.

group and the results of the ANOVA test are shown in Table 4. The Em was significantly lower and the peak early diastolic filling velocity to Em ratio (E/Em) was significantly higher in nonsurvivors compared to survivors. The area under the ROC curve was 0.93 for Em and 0.89 for the ratio E/Em. A cut-off value of $0.177 \mathrm{~m} / \mathrm{s}$ for Em predicted mortality with $100 \%$ sensitivity and $73 \%$ specificity $(Y=0.72)$. A cut-off value of 2.67 for E/Em predicted mortality with $100 \%$ sensitivity and $83 \%$ specificity $(Y=0.83$; Figure 1$)$.

Other diastolic parameters including peak early diastolic LV filling velocity (E), E deceleration time (DTE), peak late diastolic LV filling velocity (A), E to A ratio (E/A) and IVRT were not significantly different between the 2 groups.

\section{Discussion}

Our results suggest that in horses with colic and naturally-occurring SIRS, those patients which succumb or are euthanized show more pronounced LV systolic and diastolic dysfunction compared to those which survive. Certain markers of systolic (SI, ET, ETI, PEP/ET) or diastolic $(\mathrm{Em}, \mathrm{E} / \mathrm{Em})$ function emerged as valuable predictors of mortality in these cases. As the SS based mainly on HR and blood lactate concentration permits to establish a prognosis in colic horses on admission, ${ }^{6}$
Table 3: Comparison of the means \pm SD of echocardiographic parameters of global and systolic function derived from pulse wave Doppler-mode in 12 surviving and 29 nonsurviving horses admitted for colic complicated by systemic inflammatory response syndrome. Least-squares-mean test was significant if $P<0.05$ (ANOVA model including breed, body weight, age, sex, and outcome

\begin{tabular}{|c|c|c|c|}
\hline Outcome & $\begin{array}{l}\text { Survivors } \\
n=12\end{array}$ & $\begin{array}{l}\text { Non survivors } \\
n=29\end{array}$ & $P$-value \\
\hline VTI & $21.57 \pm 5.99$ & $15.86 \pm 7.78$ & 0.0061 \\
\hline SV (mL) & $1027.53 \pm 322.23$ & $720.74 \pm 299.30$ & 0.0055 \\
\hline SI (mL/kg) & $2.079 \pm 0.664$ & $1.491 \pm 0.637$ & 0.0048 \\
\hline $\mathrm{CO}(\mathrm{L} / \mathrm{mn})$ & $50.96 \pm 14.4$ & $46.6 \pm 13.42$ & 0.3339 \\
\hline $\mathrm{Cl}(\mathrm{mL} / \mathrm{kg} / \mathrm{mn})$ & $103.52 \pm 31.93$ & $96.51 \pm 28.74$ & 0.307 \\
\hline $\operatorname{Vmax}(\mathrm{m} / \mathrm{s})$ & $0.833 \pm 0.16$ & $0.804 \pm 0.161$ & 0.5692 \\
\hline TTP (ms) & $132.87 \pm 41.29$ & $103.61 \pm 36.33$ & 0.0617 \\
\hline $\mathrm{dv} / \mathrm{dt}\left(\mathrm{m} / \mathrm{s}^{2}\right)$ & $7.23 \pm 3.31$ & $9.45 \pm 5.31$ & 0.2875 \\
\hline $\mathrm{Ddv} / \mathrm{dt}\left(\mathrm{m} / \mathrm{s}^{2}\right)$ & $3.5 \pm 0.73$ & $4.15 \pm 1.25$ & 0.2316 \\
\hline DT (ms) & $248.43 \pm 36.27$ & $203.15 \pm 48.47$ & 0.0197 \\
\hline $\operatorname{PEP}(\mathrm{ms})$ & $62.15 \pm 25.84$ & $68.84 \pm 26.07$ & 0.2986 \\
\hline ET (ms) & $370.67 \pm 66.91$ & $302.54 \pm 73.43$ & 0.0161 \\
\hline ETI (ms) & $399.23 \pm 61.41$ & $342.33 \pm 62.83$ & 0.0207 \\
\hline PEP/ET & $0.166 \pm 0.06$ & $0.235 \pm 0.102$ & 0.0222 \\
\hline $\operatorname{Vcf}(\mathrm{mm} / \mathrm{s})$ & $1.075 \pm 0.28$ & $1.394 \pm 0.506$ & 0.0434 \\
\hline
\end{tabular}

CO: cardiac output; $\mathrm{Cl}$ : cardiac index; $\mathrm{dv} / \mathrm{dt}$ : acceleration rate of aortic flow ejection phase; Ddv/dt: deceleration rate of aortic flow ejection phase; DT: deceleration time of aortic ejection phase; ET: aortic flow ejection time; ETI: ejection time index (corrected for HR) $=\mathrm{ET}+(0.55 * \mathrm{FC})$; n: number of horses; PEP: left ventricular pre-ejection-period; PEP/ET: aortic flow preejection period to ejection time ratio; SI: stroke index = SV/body weight; SV: left ventricular stroke volume derived from aortic flow in Doppler-mode; TTP: time to peak = acceleration time of aortic flow ejection phase; Vcf: mean velocity of circumferential fiber shortening; Vmax: maximal velocity of the aortic flow; VTI: velocity time integral of aortic flow.

echocardiography could be used to predict more acurately the chances of survival of already shocked horses presented with tachycardia and hyperlactatemia.

A previous echocardiographic study in horses admitted for colic signs suggested that systolic dysfunction occurs with endotoxic shock; however, the results were confused by concurrent tachycardia and a drastic drop of $\mathrm{EDV}^{24}$ which can both mimic systolic ${ }^{29,32}$ as well as diastolic dysfunction. ${ }^{33}$ In the present study, all cases were selected because of the presence of SIRS. Therefore, they all exhibited a high HR and 2D and TM-mode echocardiographic evidence of a drastically low LV preload as indicated by a very low EDV compared to normal values in euhydrated horses or even experimentally hypohydrated horses. ${ }^{34}$ Since HR or EDV were not significantly different between surviving and nonsurviving horses in the present study, tachycardia and falling preload were assumed not to have greatly influenced outcome.

Although EDV is recognized as one of the best preload estimates in people with early septic shock, ${ }^{35,36}$ the frequent systolic dysfunction and dynamic changes in LV compliance associated with septic shock preclude the 


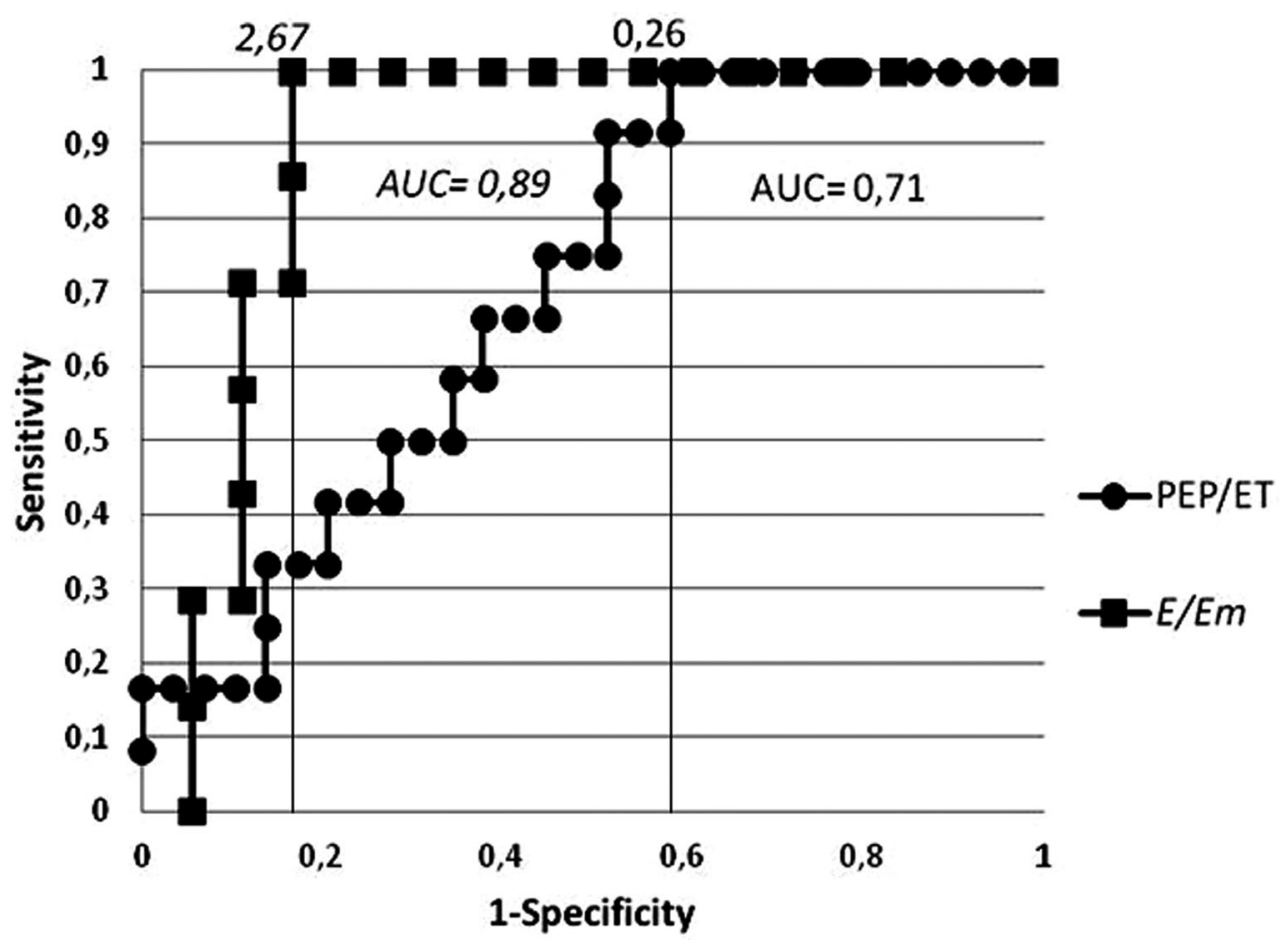

Figure 1: Receiver operating characteristic curves of PEP/ET and E/Em to predict hospital mortality in 41 horses admitted for colic complicated by systemic inflammatory response syndrome. AUC: area under the receiver operating characteristic curve; E/Em: peak early transmitral flow velocity to peak early diastolic myocardial velocity ratio; PEP/ET: aortic flow pre-ejection period to ejection time ratio.

use of EDV to predict fluid responsiveness in these patients unless LV size is very small (preload dependent patients). ${ }^{37,38}$

While indicating a favorable prognosis in people with septic shock by predicting a better response to fluid resuscitation, ${ }^{5,11}$ in the present study a low SI $(<1.78 \mathrm{~mL} / \mathrm{kg})$ on admission predicted an unfavorable outcome ( $83 \%$ sensitivity, $62 \%$ specificity). This discrepancy could be due to impaired myocardial contractility which may worsen the influence of reduced preload in nonsurviving horses, or may be due to the large volumes of fluid required by adult horses compared to people because of body size. Therefore, any preload-dependent systolic impairement is less likely to be corrected in adult horses. $^{7}$

Systolic time intervals are known to be more reliable indices of systolic function than Vcf or EF. ${ }^{39}$ However, the changes observed in this study may also be due to tachycardia or falling preload, ${ }^{29,39}$ as has been described in hemodialyzed human patients. ${ }^{32}$ Considering the comparable HR and preloading conditions in both groups, the lower ET with a higher PEP/ET ratio in the nonsurvivors may indicate a more compromised systolic function compared to surviving horses.

An increased PEP/ET ratio (>0.26) predicted mortality in colic horses with a high sensitivity $(100 \%)$ but a low specificity $(42 \%)$ in the present study. Since PEP and ET change in opposite directions with systolic dysfunction, $\mathrm{PEP} / \mathrm{ET}$ is known to be more sensitive for predicting systolic dysfunction than either of these parameters alone. ${ }^{20}$ In an early study in people with sepsis, a high PEP/ET on admission also carried a poor prognosis, ${ }^{40}$ and this ratio could be used as in human ICUs to identify horses with severe LV systolic dysfunction and a high risk of death.

Concerning diastolic function, impaired relaxation was suspected in both groups, based on the mitral flow profile with a low E/A ratio combined with a prolonged IVRT compared to previously published reference values in adult horses. ${ }^{28,30}$ This pattern may however simply result from the reduced preload ${ }^{22}$ or from the tachycardia ${ }^{33}$ observed in both groups. Indeed, in people with septic shock, reversal of the transmitral flow pattern has been attributed to falling preload and did not carry a poor prognosis unless combined with a poor LV systolic function. ${ }^{22}$

On the contrary, a low Em $(<0.177 \mathrm{~m} / \mathrm{s})$ predicted mortality with $100 \%$ sensitivity and $73 \%$ specificity in the present study, suggesting a more compromised relaxation in nonsurviving horses. Em, which reflects relaxation kinetics, ${ }^{33}$ is typically reduced in case of impaired LV relaxation associated with septic shock in people, ${ }^{41}$ 
Table 4: Comparison of the means \pm SD of echocardiographic parameters of diastolic function derived from pulse wave Dopplermode and pulse wave tissue Doppler imaging mode in 12 surviving and 29 nonsurviving horses admitted for colic complicated by systemic inflammatory response syndrome. Least-squares-mean test was significant if $P<0.05$ (ANOVA model including breed, body weight, age, sex, and outcome)

\begin{tabular}{lccl}
\hline Outcome & $\begin{array}{l}\text { Survivors } \\
\boldsymbol{n}=\mathbf{1 2}\end{array}$ & $\begin{array}{l}\text { Non survivors } \\
\boldsymbol{n}=\mathbf{2 9}\end{array}$ & $\boldsymbol{P}$-value \\
\hline $\mathrm{E}(\mathrm{m} / \mathrm{s})$ & $0.555 \pm 0.13$ & $0.571 \pm 0.193$ & 0.686 \\
VTIE & $9.4 \pm .3 .25$ & $9.03 \pm 4.94$ & 0.9964 \\
$\mathrm{dE}(\mathrm{ms})$ & $259.92 \pm 51.39$ & $230.42 \pm 87.35$ & 0.5355 \\
$\mathrm{DTE}(\mathrm{ms})$ & $141.32 \pm 48.02$ & $122.02 \pm 51.68$ & 0.6982 \\
$\mathrm{Ddv} / \mathrm{dtE}\left(\mathrm{m} / \mathrm{s}^{2}\right)$ & $4.61 \pm 1.94$ & $5.52 \pm 2.81$ & 0.4166 \\
$\mathrm{~A}(\mathrm{~m} / \mathrm{s})$ & $0.493 \pm 0.14$ & $0.579 \pm 0.2$ & 0.2397 \\
VTIA & $5.53 \pm 1.82$ & $6.46 \pm 2.43$ & 0.2464 \\
$\mathrm{dA}(\mathrm{ms})$ & $171.58 \pm 31.78$ & $174.65 \pm 41.19$ & 0.6146 \\
E/A & $1.149 \pm 0.416$ & $1.062 \pm 0.373$ & 0.4912 \\
$\mathrm{IVRT}(\mathrm{ms})^{*}$ & $83.61 \pm 30.96$ & $69.33 \pm 26.68$ & 0.1938 \\
Em $(\mathrm{m} / \mathrm{s})^{*}$ & $0.234 \pm 0.034$ & $0.135 \pm 0.057$ & 0.0010 \\
E/Em $^{*}$ & $2.08 \pm 0.42$ & $5.11 \pm 2.49$ & 0.0051 \\
\hline
\end{tabular}

*Data from pulsed-wave tissue Doppler mode were available for only 25 horses ( 7 surviving and 18 nonsurviving horses); A: peak late LV filling velocity; dA: late $L V$ filling duration; Ddv/dtE: deceleration rate of early LV filling; dE: early LV filling duration; DTE: deceleration time of early LV filling; E: peak early LV filling velocity; E/A: peak early to late LV filling velocity ratio; Em: peak early diastolic myocardial velocity; E/Em: peak early LV filling velocity to peak early diastolic myocardial velocity ratio; IVRT: isovolumic relaxation time; LV: left ventricular; VTI: velocity time integral.

and is considered to be preload independent in case of impaired relaxation. ${ }^{38,42}$ However, a mixed pattern combining impaired relaxation with restrictive ventricular filling was suspected based on a higher E/Em ratio in the nonsurviving group with a cut-off value of 2.67 offering the best sensitivity $(100 \%)$ and specificity $(83 \%)$ to predict mortality. By correcting the effect of $\mathrm{LV}$ relaxation on $\mathrm{E}$ velocity, ${ }^{38}$ the $\mathrm{E} / \mathrm{Em}$ ratio is considered one of the best Doppler estimates of LV filling pressure. ${ }^{38,42}$ Despite being observed less frequently than impaired myocardial relaxation, this restrictive filling pattern has been described in human septic patients, ${ }^{12,43}$ where E/Em recently proved to be an independent predictor of survival, even demonstrating a higher sensitivity $(100 \%)$ and specificity $(83 \%)$ than blood cardiac biomarkers or systolic function parameters. ${ }^{12}$ The origin of this sepsisinduced diastolic dysfunction is currently incompletely understood but has been associated with histological evidence of inflammatory infiltrates, interstitial edema, apoptosis and necrosis of the myocardium resulting in decreased compliance and subsequent LV pressure elevation. ${ }^{12,43}$

The main limitation of this study was related to the use of echocardiography itself, which typically provides a noninvasive assessment of LV systolic and diastolic function but is highly dependent on loading conditions of the heart and HR, which are substantially altered by SIRS. In addition, afterload was not assessed in this study. Invasive direct measurements of mean arterial pressure and central venous pressure to determine systemic vascular resistance could have provided a more reliable interpretation of our results. Even if assessment of Em by PWTDI at the level of the LV free wall in short axis has been shown to be reliable and repeatable in horses, ${ }^{28}$ Doppler parameters may be subject to variability and a follow-up of individual horses, especially the response to a fluid challenge may provide more accurate prognostic indicators than isolated Doppler measurements.

The limited number of horses included in this study, especially in the surviving group, limited its statistical power. However, the survival rate in this study (12/41 or $29.3 \%$ ) is in agreement with previous data of horses with colic complicated by SIRS. ${ }^{6}$

In the absence of a well-established definition in horses, the diagnosis of SIRS in this study was based on clinical and blood parameters mirroring the defining criteria of SIRS in human medicine ${ }^{9,44}$ associated with hyperlactatemia or low systolic arterial pressure as markers of hypoperfusion without attempting to identify endotoxins in the blood. Nevertheless, the detection of lipopolysaccharides in the blood suffered from low sensitivity (only $58.4 \%$ ) and specificity $(87.5 \%$ ) to detect clinical endotoxemia and added little to the prognosis of equine colic compared to clinical parameters. ${ }^{45}$

The underlying mechanisms of sepsis-induced myocardial failure are not well understood, but several pathways have been hypothesized in human patients. They include excess production of nitric oxide in cardiomyocytes with direct effect on contractility and via a cytopathic hypoxia as a consequence of depressed mitochondrial respiration and impaired oxygen utilization. ${ }^{4,5,46}$ This hypoxia could be worsened by an impairment of auto-regulating mechanisms of microcirculation, which generates a heterogenous myocardial oxygen supply. ${ }^{47}$ Furthermore, endotoxins disrupt calcium transfer at various sites, causing a decrease in calcium sensitivity and an unresponsiveness to $\beta$ adrenergic stimulation of the cardiomyocytes. ${ }^{4}$ Other factors such as autonomic nervous system dysfunction, ${ }^{5}$ myocardial inflammatory infiltration, ${ }^{46}$ or a tachycardiainduced cardiomyopathy ${ }^{4}$ may similarly contribute to myocardial depression. All or some of these factors could result in a decrease in myocardial contractility and compliance. ${ }^{4,5}$

Therefore, cardiac performance may be altered by impairement of either diastolic function (ventricular filling defect secondary to combined low venous return and 
impaired relaxation or compliance) or systolic function (lack of contractility). Decreased cardiac performance with low EF and SV could be transiently compensated by sympathic stimulation and tachycardia to maintain $\mathrm{CO}$ but may result in circulatory failure with a drop of $\mathrm{CO}$ (eg, cardiogenic shock). In the present study, CI showed a high interindividual variability in nonsurviving horses, and this could be due to different hemodynamic profiles mirroring those observed in human patients who succumb from septic shock. ${ }^{11,36,38}$ This way, a cardiogenic shock with low CI was suspected in 3 horses (including the one spontaneous death) although a classic distributive shock characterized by refractory hypotension despite a preserved CI (with late appearance of MODS in some cases) was recognized in most horses subjected to euthanasia.

This myocardial dysfunction (systolic or diastolic) is known to be reversible within 7 to 10 days in human patients who survive septic shock. ${ }^{4}$ This phenomenon is sometimes considered a protective mechanism to reduce energy expenditure and oxygen demand during endotoxic shock. This "cardiac hibernation" presumably allows preservation of heart viability and integrity. ${ }^{4} \mathrm{Al}-$ though an excellent recovery of cardiac performance has been observed in most surviving patients, some children suffered from long-term cardiac complications such as exercise-induced arrythmia or decreased LV function after septic shock. ${ }^{48}$ The athletic prognosis and any delayed effects of SIRS on cardiac function are currently unknown in horses and warrants further investigation.

\section{Conclusion}

In the present study, certain echocardiographic markers of either systolic (PEP/ET > 0.26) or diastolic $(\mathrm{E} / \mathrm{Em}>2.67)$ dysfunction predicted a poor outcome in colic horses with SIRS, suggesting that echocardiography may provide useful prognostic information in these horses as has been shown in human patients with septic shock. ${ }^{12,20}$ The clinical significance of the more marked myocardial depression occuring with SIRS in nonsurviving horses warrants further investigation. Early use of hemodynamic monitoring during the course of severe sepsis and septic shock with the implementation of early goal directed therapy has significantly lowered mortality in human ICUs. ${ }^{2,44}$ As already used in people, ${ }^{2,8,11,12,17}$ echocardiography could further be used in equine critical care to assess the response to fluid delivery and to guide early selection of appropriate support treatments (ie, fluids, positive inotropes, vasopressors).

\section{Footnotes \\ a Accutrend Roche Diagnostics, Mannheim, Germany \\ b Medonic, CA 530 Oden, Sweden.}

Dinamap ${ }^{\circledR} 845$ Veterinary Blood Pressure Monitoring, Critikon, Tampa, FL.

d Vivid I, General Electric, Diegem, Belgium.

e Echopac, General Electric Healthcare Europe GMBH., Diegem, Belgium.

SAS Institute Inc., Cary, NC.

\section{References}

1. Ward DS, Fessler JF, Bottoms GD, et al. Equine endotoxemia: cardiovascular, eicosanoid, hematologic, blood chemical, and plasma enzyme alterations. Am J Vet Res 1987; 7:1150-1156.

2. Casserly B, Read R, Levy MM. Hemodynamic monitoring in sepsis. Crit Care Clin 2009; 25:803-823.

3. Roy MF. Sepsis in adults and foals. Vet Clin North Am Equine Pract 2004; 20:41-61.

4. Rudiger A, Singer M. Mechanisms of sepsis-induced cardiac dysfunction. Crit Care Med 2007; 35:1599-1608.

5. Zanotti-Cavazonni SL, Hollenberg SM. Cardiac dysfunction in severe sepsis and septic shock. Curr Opin Crit Care 2009; 15:392-397.

6. Grulke S, Olle E, Detilleux J, et al. Determination of a gravity and shock score for prognosis in equine surgical colics. J Vet Med 2001; A48:465-473.

7. Corley KTT. Inotropes and vasopressors in adults and foals. Vet Clin North Am Equine Pract 2004; 20:77-106.

8. Boyd JH, Walley KR. The role of echocardiography in hemodynamic monitoring. Curr Opin Crit Care 2009; 15:239-243.

9. Jones AE, Saak K, Kline JA. Performance of the mortality in emergency department sepsis score for predicting hospital mortality among patients with severe sepsis and septic shock. Am J Emerg Med 2008; 26(6):689-692.

10. Carpenter CR, Keim SM, Upadhye S, Nguyen HB. Risk stratification of the potentially septic patient in the emergency department: the mortality in the emergency department sepsis (MEDS) score. J Emerg Med 2009; 37(3):319-327.

11. Court O, Kumar A, Parillo JE, et al. Clinical review: myocardial depression in sepsis and septic shock. Crit Care 2002; 6(6):500-508.

12. Sturgess DJ, Marwick TH, Joyce C, et al. Prediction of hospital outcome in septic shock: a prospective comparison of tissue Doppler and cardiac biomarkers. Crit Care 2010; 14(2):R44 doi:1186/cc8931.

13. Kumar A, Schupp E, Bunnell E, et al. Cardiovascular response to dobutamine stress predicts outcome in severe sepsis and septic shock. Crit Care 2008; 12(R35):doi:10.1186/cc6814.

14. Jones AE, Shapiro NL, Trzeciak S, et al. Lactate clearance vs central venous oxygene saturation as goals of early sepsis therapy. J Am Med Assoc 2010; 303(8):739-746.

15. Tennent-Brown BS, Wilkins PA, Lindborg S, et al. Sequential plasma lactate concentrations as prognostic indicators in adult equine emergencies. J Vet Intern Med 2010; 24:198-205.

16. Hashimoto-Hill S, Magdesian KG, Kass PH. Serial measurement of lactate concentration in horses with acute colitis. J Vet Intern Med $2011 ; 25: 1414-1419$.

17. Hollenberg SM. Inotrope and vasopressor therapy of septic shock. Crit Care Clin 2009; 25:781-802.

18. Rosjo H, Varpula M, Agve TA, et al. Circulating high sensitivity tropinin $\mathrm{T}$ in severe sepsis and septic shock: distribution, associated factors and relation to outcome. Intensive Care Med 2011; 37(1):7785.

19. Januzzi JL, Morss A, Tung R, et al. Natriuretic peptide testing for the evaluation of critically ill patients with shock in the intensive care unit: a prospective cohort study. Crit Care 2006; 10(1):R37 doi:10.1186/cc4839.

20. Artucio H, Digenio A, Pereyra M. Left ventricular function during sepsis. Crit Care Med 1989; 17:323-327.

21. Bouhemad B, Nicolas-Robin A, Arbelot C, et al. Isolated and reversible impairement of ventricular relaxation in patients with septic shock. Crit Care Med 2008; 36:766-774.

22. Poelaert J, Declerck C, Vogelaers D, et al. Left ventricular systolic and diastolic function in septic shock. Intensive Care Med 1997; 23:553-560.

23. Radcliffe RM, Divers TJ, Fletcher DJ, et al. Evaluation of L-lactate and cardiac tropinin I in horses undergoing emergency abdominal surgery. J Vet Emerg Crit Care 2012; 22(3):313-319. 
24. Borde L, Amory H, Leroux A, et al. Echocardiographic assessment of left ventricular function in colic horses. J Equine Vet Science 2011; 31(8):481-487.

25. Boon JA. The two-dimensional echocardiographic exam. In: Boon JA. ed. Veterinary echocardiography. 2nd edn. Ames, IA: WileyBlackwell; 2011, pp. 527-581.

26. Boon JA. The M-Mode and Doppler examination. In: Boon JA. ed. Veterinary echocardiography. $2^{\text {nd }}$ edn. Ames, IA: Wiley-Blackwell; 2011, pp. 101-151.

27. Boon JA. Evaluation of size, function, and hemodynamics. In: Boon JA. ed. Veterinary echocardiography. $2^{\text {nd }}$ edn. Ames, IA: WileyBlackwell; 2011, pp. 153-256.

28. Schwarzwald CC, Shober KE, Bonagura JD. Methods and reliability of tissue Doppler imaging for assessment of left ventricular radial wall motion in horses. J Vet Intern Med 2009; 23:643-652.

29. Lightlower C, Piccone G, Fazio F, et al. Systolic time intervals assessed by 2-D echocardiography and spectral Doppler in the horse. An Sci J 2003; 74:505-510.

30. Blissit KJ, Young LE, Jones RS, Drake PGG, Utting J. Measurement of cardiac output in standing horses by Doppler echocardiography and thermodilution. Equine Vet J 1997; 29(1):18-25.

31. Young LE, Scott R. Measurement of cardiac function by transthoracic echocardiography: day to day variability and repeatability in normal thoroughbred horses. Equine Vet J 1998; 30:117122.

32. Ouali S, Abroug S, Neffeti E, et al. Effects of acute decrease in preload on echocardiographic indices of systolic and diastolic function of the left ventricule in children with end-stage renal disease (ERSD). Comparative study before and after hemodialysis. Ann Cardiol Angeiol 2010; 59(1):14-19.

33. Nagueh SF, Appleton CP, Gillebert TC, et al. Recommendations for the evaluation of left ventricular dysfunction by echocardiography. J Am Soc Echocardiography 2009; 22(2):107-133.

34. Underwood C, Norton JL, Nolen-Walston RD, et al. Echocardiographic changes in heart size in hypohydrated horses. J Vet Intern Med 2011; 25(3):563-569

35. Scheuren K, Wente MN, Hainer C, et al. Left ventricular enddiastolic area is a measure of cardiac preload in patients with early septic shock. Eur J Anaesthesiol 2009; 26:759-765.
36. Osman D, Ridel C, Ray P, et al. Cardiac filling pressures are not appropriate to predict hemodynamic response to volume challenge. Crit Care Med 2007; 35:64-68.

37. Levitov A, Marik PE. Echocardiographic assessment of preload responsiveness in critically ill patients. Cardiology Res Pract 2012, doi:10.1155/2012/819696.

38. Lamia B, Ochagavia A, Monnet X, et al. Echocardiographic prediction of volume responsiveness in critically ill patients with spontaneously breathing activity. Intensive Care Med 2007; 33:1125-1132.

39. Thomas JD, Popovic ZB. Assessment of left ventricular function by cardiac ultrasound. J Am Coll Cardiol 2006; 48:2012-2025.

40. Kumar A, Haery C, Parillo JE. Myocardial dysfunction in septic shock. Crit Care Clin 2000; 16:251-287.

41. Etchecopar-Chevreuil C, François B, Clavel M, et al. Cardiac morphological and functional changes during early septic shock: a transesophageal echocardiographic study. Intensive Care Med 2008 34:250-256.

42. Mousavi N, Czarnecki A, Ahmadie R, et al. The utility of tissue Doppler imaging for the non invasive determination of left ventricular filling pressures in patients with septic shock. J Intensive Care Med 2010; 25(3):163-167.

43. Bouhemad B, Nicolas-Robin A, Arbelot C, et al. Acute left ventricular dilatation and shock-induced myocardial dysfunction. Crit Care Med 2009; 37:441-447.

44. Puskarich MA, Marchick MR, Kline JA, et al. One year mortality of patients treated with an emergency department based early goal directed therapy protocol for severe sepsis and septic shock: a before and an after study. Crit Care 2009; 13:R167 doi:10.1186/cc8138.

45. Senior JM, Proudman CJ, Leuwer M, Carter SD. Plasma endotoxin in horses presented to an equine referral hospital: correlation to selected clinical parameters and outcomes. Equine Vet J 2011 43(5):581-591.

46. Merx MW, Werber C. Sepsis and the heart. Circulation 2007; 116:793802.

47. Ellis CG, Jagger J, Sharpe M. The microcirculation as a functional system. Crit Care 2005; 9(suppl 4):S3-S8 doi:10.1186/cc3751.

48. Knoester $\mathrm{H}$, Sol JJ, Ramsodit $\mathrm{P}$, et al. Cardiac function in pediatric septic shock survivors. Arch Pediatr Adolesc Med 2008, 162(12):1164-1168 